Corrected Draft 08 August 2006

\title{
The self-energy of the uniform electron gas in the second order of exchange
}

\author{
P. ZIESCHE \\ Max-Planck-Institut für Physik komplexer Systeme \\ Nöthnitzer Str. 38, D-01187 Dresden, Germany \\ pz@pks.mpg.de
}

PACS 71.10.Ca, 05.30.Fk

\begin{abstract}
The on-shell self-energy of the homogeneous electron gas in second order of exchange, $\Sigma_{2 \mathrm{x}}=\operatorname{Re} \Sigma_{2 \mathrm{x}}\left(k_{\mathrm{F}}, k_{\mathrm{F}}^{2} / 2\right)$, is given by a certain integral. This integral is treated here in a similar way as Onsager, Mittag, and Stephen [Ann. Physik (Leipzig) 18, 71 (1966)] have obtained their famous analytical expression $e_{2 \mathrm{x}}=\frac{1}{6} \ln 2-\frac{3}{4} \frac{\zeta(3)}{\pi^{2}}$ (in atomic units) for the correlation energy in second order of exchange. Here it is shown that the result for the corresponding on-shell self-energy is $\Sigma_{2 \mathrm{x}}=e_{2 \mathrm{x}}$. The off-shell self-energy $\Sigma_{2 \mathrm{x}}(k, \omega)$ correctly yields $2 e_{2 \mathrm{x}}$ (the potential component of $e_{2 \mathrm{x}}$ ) through the Galitskii-Migdal formula. The quantities $e_{2 \mathrm{x}}$ and $\Sigma_{2 \mathrm{x}}$ appear in the high-density limit of the Hugenholtz-van Hove (Luttinger-Ward) theorem.
\end{abstract}

Typeset by REVTEX 


\section{INTRODUCTION}

Although not present in the Periodic Table the homogeneous electron gas (HEG) is still an important model system for electronic structure theory, cf. e.g. 1]. In its spin-unpolarized version the HEG ground state is characterized by only one parameter $r_{s}$, such that a sphere with the radius $r_{s}$ contains on average one electron [2]. It determines the Fermi wave number as $k_{\mathrm{F}}=1 /\left(\alpha r_{s}\right)$ with $\alpha=(4 /(9 \pi))^{1 / 3}$ and it measures simultaneously the interaction strength and the density such that high density corresponds to weak interaction and hence weak correlation. For recent papers on this limit cf. [3, 4, 5]. Naively one should expect that in this weak-correlation limit the Coulomb repulsion $\alpha r_{s} / r$ (where lengths and energies are measured in units of $k_{\mathrm{F}}^{-1}$ and $k_{\mathrm{F}}^{2}$, respectively) can be treated as perturbation. But in the early theory of the HEG, Heisenberg [6] has shown, that ordinary perturbation theory does not apply. With $e_{0}$ being the energy per particle of the ideal Fermi gas and $e_{\mathrm{x}}$ being the exchange energy in lowest (1st) order, the total energy $e=e_{0}+e_{\mathrm{x}}+e_{\mathrm{c}}$ defines the correlation energy $e_{\mathrm{c}}=e_{2}+e_{3}+\cdots$. In 2nd order, there is a direct $(\mathrm{d})$ term $e_{2 \mathrm{~d}}$ and an exchange $(\mathrm{x})$ term $e_{2 \mathrm{x}}$, so that $e_{2}=e_{2 \mathrm{~d}}+e_{2 \mathrm{x}}$. Whereas $e_{2 \mathrm{x}} /\left(\alpha r_{s}\right)^{2}$ is a pure finite number $b_{\mathrm{x}}$, the direct term $e_{2 \mathrm{~d}}$ logarithmically diverges along the Fermi surface (i.e. for vanishing transition momenta $q$ ): $e_{2 \mathrm{~d}} \rightarrow \ln q$ for $q \rightarrow 0$. This failure of perturbation theory has been repaired by Macke 7] with an appropriate partial summation of higher-order terms up to infinite order. The result of this (ring-diagram) summation for the correlation energy in its weak-correlation limit is $e_{\mathrm{c}} /\left(\alpha r_{s}\right)^{2}=a \ln r_{s}+\cdots$ with $a=(1-\ln 2) / \pi^{2} \approx 0.031091$. This has been confirmed later by Gell-Mann and Brueckner [8], who in addition to the logarithmic term numerically calculated contributions to the next (constant, i.e. not depending on $r_{s}$ ) term $b$, namely $b_{\mathrm{r}}$ and $b_{\mathrm{d}}$ arising from the ring-diagram summation and from $e_{2 \mathrm{~d}}$, respectively: $b_{\mathrm{r}} \approx a \ln \frac{\alpha}{\pi}-0.001656 \approx-0.057514$ and $b_{\mathrm{d}} \approx-0.013586$. The total constant term is $b=b_{\mathrm{r}}+b_{\mathrm{d}}+b_{\mathrm{x}} \approx-0.046921$, where the

exchange term $b_{\mathrm{x}}=\frac{1}{6} \ln 2-\frac{3}{4} \frac{\zeta(3)}{\pi^{2}} \approx+0.0241792$ has been analytically calculated by Onsager, Mittag, and Stephen in a very tricky way [9]. Although the integrand is rather simple, the Pauli principle causes complicated boundary conditions, which need a sophisticated treatment through several substitutions of the integral variables.

Here their method is used to calculate the analog term of the self-energy on the energy shell, 
namely $\Sigma_{2 \mathrm{x}}=\operatorname{Re} \Sigma_{2 \mathrm{x}}(1,1 / 2)$ [with $k$ measured in units of $k_{\mathrm{F}}$ and $\omega$ measured in units of $k_{\mathrm{F}}^{2}$ ]. The more general quantity $\Sigma_{2 \mathrm{x}}(k, \omega)$ appears (i) in a recent study of the spectral moments of the HEG [11], (ii) in the context of self-consistent $G W$ calculations [12], and within a project 'spectral functions for a hydrogen plasma' 13]. For $k=1, \omega=\mu$ it appears in the Hugenholtz-van Hove (Luttinger-Ward) identity [14], which relates the chemical potential $\mu$ to the self-energy $\Sigma(k, \omega)$ according to $\mu-\mu_{0}=\Sigma(1, \mu)$. The chemical potential follows from the total energy according to $\mu=\left(\frac{5}{3}-\frac{1}{3} r_{s} \frac{d}{d r_{s}}\right) e$. In the weak-correlation limit $r_{s} \rightarrow 0$ the total energy $e=e_{0}+e_{\mathrm{x}}+e_{\mathrm{c}}$ and the chemical potential $\mu=\mu_{0}+\mu_{\mathrm{x}}+\mu_{\mathrm{c}}$ are given by

$$
\begin{gathered}
e_{0}=\frac{3}{10}, \quad e_{\mathrm{x}}=-\frac{3}{4} \frac{\alpha r_{s}}{\pi}, \quad e_{\mathrm{c}}=\left(\alpha r_{s}\right)^{2}\left[a \ln r_{s}+b+O\left(r_{s}\right)\right], \\
\mu_{0}=\frac{1}{2}, \quad \mu_{\mathrm{x}}=-\frac{\alpha r_{s}}{\pi}, \quad \mu_{\mathrm{c}}=\left(\alpha r_{s}\right)^{2}\left[a \ln r_{s}+\left(-\frac{1}{3} a+b\right)+O\left(r_{s}\right)\right] .
\end{gathered}
$$

Because of the above mentioned identity the self-energy $\Sigma(1, \mu)$ must have a corresponding behavior. The exchange in lowest order yields $\Sigma_{\mathrm{x}}(1)=-\frac{\alpha r_{s}}{\pi}$ or $\mu_{\mathrm{x}}=\Sigma_{\mathrm{x}}(1)$, exactly in agreement with the mentioned identity. To obtain also the logarithmic term of $\Sigma(1,1 / 2)$ the ring-diagram summation has to be done for the self-energy [15]. To the next term beyond the logarithmic term contributes the 2nd-order exchange self-energy $\Sigma_{2 \mathrm{x}}=\operatorname{Re} \Sigma_{2 \mathrm{x}}(1,1 / 2)$. Just this term is calculated here using the tricky method of Onsager, Mittag, and Stephen 9] mutatis mutandi. The Feynman diagrams of $e_{2 \mathrm{x}}$ and $\Sigma_{2 \mathrm{x}}$ are given in Figs. 1 and 2. As shown in the Appendix, from the Feynman diagram rules it follows $\Sigma_{2 \mathrm{x}}=-\frac{\left(\alpha r_{s}\right)^{2}}{4 \pi^{4}}\left(X_{1}+X_{2}\right)$, where $X_{1,2}$ mean the integrals defined in Eqs. A.10 and (A.11). They are calculated in the following sections. The final results are $X_{1}=-\pi^{4}\left[\frac{4}{3} \ln 2-5 \frac{\zeta(3)}{\pi^{2}}\right], X_{2}=\pi^{4}\left[\frac{2}{3} \ln 2-2 \frac{\zeta(3)}{\pi^{2}}\right]$, thus $\Sigma_{2 \mathrm{x}}=\left(\alpha r_{s}\right)^{2}\left[\frac{1}{6} \ln 2-\frac{3}{4} \frac{\zeta(3)}{\pi^{2}}\right]=e_{2 \mathrm{x}}$, thus $\mu_{2 \mathrm{x}}=\Sigma_{2 \mathrm{x}}$. This relation appears in the weakcorrelation limit of the above mentioned Hugenholtz-van Hove theorem 14, 15]. This paper is a contribution to the mathematics of the weakly-correlated (high-density) HEG.

\section{THE INTEGRAL $X_{1}$}

In Eq. (A.10), new variables $\boldsymbol{q}^{\prime}=\left(\boldsymbol{q}_{1}+\boldsymbol{q}_{2}\right) / 2, \boldsymbol{p}^{\prime}=\left(\boldsymbol{q}_{1}-\boldsymbol{q}_{2}\right) / 2$, and $\boldsymbol{s}^{\prime}=\boldsymbol{e}+\left(\boldsymbol{q}_{1}+\boldsymbol{q}_{2}\right) / 2$ lead to

$$
\begin{aligned}
& \boldsymbol{q}_{1}=\boldsymbol{q}^{\prime}+\boldsymbol{p}^{\prime}, \quad\left(\boldsymbol{e}+\boldsymbol{q}_{1}\right)^{2}=\left(\boldsymbol{s}^{\prime}+\boldsymbol{p}^{\prime}\right)^{2}, \quad\left(\boldsymbol{e}+\boldsymbol{q}_{2}\right)^{2}=\left(\boldsymbol{s}^{\prime}-\boldsymbol{p}^{\prime}\right)^{2}, \\
& \boldsymbol{q}_{2}=\boldsymbol{q}^{\prime}-\boldsymbol{p}^{\prime}, \quad\left(\boldsymbol{e}+\boldsymbol{q}_{1}+\boldsymbol{q}_{2}\right)^{2}=\left(\boldsymbol{s}^{\prime}+\boldsymbol{q}^{\prime}\right)^{2}, \quad \boldsymbol{e}^{2}=\left(\boldsymbol{s}^{\prime}-\boldsymbol{q}^{\prime}\right)^{2} .
\end{aligned}
$$


Therefore (with $\Theta(x)=$ Heaviside step function)

$$
\begin{gathered}
X_{1}=\int d^{3} q^{\prime} d^{3} p^{\prime} \frac{2^{3}}{q^{\prime 2}-p^{\prime 2}} \frac{1}{\left(\boldsymbol{p}^{\prime}+\boldsymbol{q}^{\prime}\right)^{2}\left(\boldsymbol{p}^{\prime}-\boldsymbol{q}^{\prime}\right)^{2}} \times \\
\times \int \frac{d^{2} s^{\prime}}{\pi} \delta\left(\left(\boldsymbol{s}^{\prime}-\boldsymbol{q}^{\prime}\right)^{2}-1\right) \Theta\left[1-\left(\boldsymbol{s}^{\prime}+\boldsymbol{q}^{\prime}\right)^{2}\right] \Theta\left[\left(\boldsymbol{s}^{\prime}+\boldsymbol{p}^{\prime}\right)^{2}-1\right] \Theta\left[\left(\boldsymbol{s}^{\prime}-\boldsymbol{p}^{\prime}\right)^{2}-1\right] .
\end{gathered}
$$

The next step scales $\boldsymbol{q}^{\prime}, \boldsymbol{p}^{\prime}, \boldsymbol{s}^{\prime}$ with $\lambda=1 / \sqrt{1-s^{\prime 2}}$ according to $\boldsymbol{q}=\lambda \boldsymbol{q}^{\prime}, \boldsymbol{p}=\lambda \boldsymbol{p}, \boldsymbol{s}=\lambda \boldsymbol{s}^{\prime}$ with the consequences

$$
\begin{array}{r}
1-s^{\prime 2}=\frac{1}{1+s^{2}}, \quad d^{2} s^{\prime}=\frac{d^{2} s}{\left(1+s^{2}\right)^{2}}, \\
\delta\left(\left(\boldsymbol{s}^{\prime}-\boldsymbol{q}^{\prime}\right)^{2}-1\right)=\left(1+s^{2}\right) \delta\left(q^{2}-2 \boldsymbol{s} \boldsymbol{q}-1\right), \\
\pm 2 \boldsymbol{s} \boldsymbol{p}+p^{2}>1, \quad 2 \boldsymbol{s} \boldsymbol{q}+q^{2}<1, \quad-2 \boldsymbol{s} \boldsymbol{q}+q^{2}=1,
\end{array}
$$

from which follow $p \geq 1$ and $q \leq 1$ (what makes the energy denominator $q^{2}-p^{2}$ negative) and $\boldsymbol{s q}<0, s \geq \alpha$. Thus

$$
\begin{array}{r}
X_{1}=-\int_{0}^{1} d q \int_{1}^{\infty} d p \frac{16 \pi}{p^{2}-q^{2}} \int_{-1}^{+1} \frac{d x}{2} \frac{8 q^{2} p^{2}}{\left(p^{2}+q^{2}\right)^{2}-(2 \boldsymbol{q} \boldsymbol{p})^{2}} \times \\
\times \int_{s \geq \alpha, \cos \varphi_{q}<0} \frac{d^{2} s}{1+s^{2}} \delta\left(q^{2}-1-2 \boldsymbol{s q}\right) \Theta\left(p^{2}-1+2 \boldsymbol{s p}\right) \Theta\left(p^{2}-1-2 \boldsymbol{s p}\right) .
\end{array}
$$

Here and in the following of Sec. II the abbreviations

$$
\begin{array}{r}
\alpha=\frac{1-q^{2}}{2 q} \geq 0, \quad \beta=\frac{p^{2}-1}{2 p} \geq 0, \quad a=\frac{q^{2}+p^{2}}{2 q p} \geq 1, \\
\frac{t}{s}=\cos \varphi_{p}=\cos \Varangle(\boldsymbol{s}, \boldsymbol{p}), \quad x=\cos \varphi=\cos \Varangle(\boldsymbol{q}, \boldsymbol{p}), \quad y=\cos \varphi_{q}=\cos \Varangle(\boldsymbol{q}, \boldsymbol{s})
\end{array}
$$

are used:

$$
X_{1}=-\int_{0}^{1} d q \int_{1}^{\infty} d p \frac{16 \pi}{p^{2}-q^{2}} \int_{-1}^{+1} \frac{d x}{a^{2}-x^{2}} \int_{s \geq \alpha, \cos \varphi_{q}<0} \frac{s d s d \varphi_{q}}{1+s^{2}} \frac{1}{2 s q} \delta\left(\frac{\alpha}{s}+y\right) \Theta(\beta-|t|)
$$

$t$ is introduced to replace $s$ after having done the $\varphi_{q}$ integration. To this purpose the relation between $t$ and $s$ is needed. It follows from $\varphi_{q}+\varphi_{p}=\varphi$ or $\varphi_{p}=\varphi-\varphi_{q}$, therefore $\cos \varphi_{p}=\cos \left(\varphi-\varphi_{q}\right)=\cos \varphi \cos \varphi_{q}+\sin \varphi \sin \varphi_{q}$, what is in terms of $t / s, x$, and $y$ (the latter quantity equals $-\alpha / s$ because of the delta function):

$$
\frac{t}{s}=x\left(-\frac{\alpha}{s}\right) \pm \sqrt{1-x^{2}} \sqrt{1-\left(\frac{\alpha}{s}\right)^{2}} \quad \text { or } \quad(t+\alpha x)^{2}=\left(1-x^{2}\right)\left(s^{2}-\alpha^{2}\right) .
$$


This has the consequences

$$
\begin{array}{r}
s^{2}-\alpha^{2}=\frac{(t+\alpha x)^{2}}{1-x^{2}}, \\
\frac{s d s}{1+s^{2}}=\frac{(t+\alpha x) d t}{(t+\alpha x)^{2}+\left(1+\alpha^{2}\right)\left(1-x^{2}\right)} .
\end{array}
$$

With the help of Eq. (2.8) the $\varphi_{q}$ integration yields a function depending on $s$ (respectively on $t$ ). Note that $\cos \varphi_{q}=-\frac{\alpha}{s}$ has two solutions $\varphi_{q, 1}$ and $\varphi_{q, 2}$ with $\left|\sin \varphi_{q, 1}\right|=\left|\sin \varphi_{q, 2}\right|=$ $\sqrt{1-\left(\frac{\alpha}{s}\right)^{2}}$ :

$$
\begin{aligned}
& \int_{0}^{2 \pi} \frac{d \varphi_{q}}{s}\left[\delta\left(-\sin \varphi_{q, 1} \cdot\left(\varphi_{q}-\varphi_{q, 1}\right)\right)+\delta\left(-\sin \varphi_{q, 2} \cdot\left(\varphi_{q}-\varphi_{q, 2}\right)\right)\right] \\
= & \int_{0}^{2 \pi} \frac{d \varphi_{q}}{s} \frac{\delta\left(\varphi_{q}-\varphi_{q, 1}\right)+\delta\left(\varphi_{q}-\varphi_{q, 2}\right)}{\sqrt{1-\left(\frac{\alpha}{s}\right)^{2}}}=\frac{2}{\sqrt{s^{2}-\alpha^{2}}}=2 \frac{\sqrt{1-x^{2}}}{|t+\alpha x|} .
\end{aligned}
$$

Thus

$$
\begin{aligned}
\int_{s \geq \alpha, \cos \varphi_{q}} \frac{s d s d \varphi_{q}}{1+s^{2}} \frac{1}{2 s q} \delta\left(\frac{\alpha}{s}+y\right) \Theta(\beta-|t|) & =\frac{\sqrt{1-x^{2}}}{q} \int_{t_{0}}^{t_{1}} \frac{\operatorname{sign}(t+\alpha x) d t}{(t+\alpha x)^{2}+\left(1+\alpha^{2}\right)\left(1-x^{2}\right)} \\
& =\left.\frac{2}{1+q^{2}} \arctan \frac{t+\alpha x}{\sqrt{\left(1+\alpha^{2}\right)\left(1-x^{2}\right)}}\right|_{t_{0}} ^{t_{1}} \cdot(2.11)
\end{aligned}
$$

$|t| \leq \beta$ contains the upper limit of the $t$-integration as $t \leq \beta=: t_{1}$. What concerns its lower limit $t_{0}$, the relation $t \geq-\beta$ competes with $t \geq-\alpha x$, as it follows from Eq. (2.7) for the lower limit $\alpha$ of the $s$-integration. This means for the ranges of the $t$ - and $x$-integrations, one has in the (vertical) stripe $q=0 \cdots 1, p=1 \cdots \infty$ of the $q$-p-plane to distinguish between the two regions, cf. Fig. 3:

(a) region $\mathrm{A}$ with $q p \geq 1$ or $\alpha \leq \beta$ or $-\alpha x \geq-\alpha \geq-\beta$, hence $t_{0}=-\alpha x$, and

(b) region $\mathrm{B}$ with $q p \leq 1$ or $\alpha \geq \beta$ or $-\alpha \leq-\beta$.

In the case (b) one has again to distinguish between

(i) $x=-\beta / \alpha \cdots+\beta / \alpha$, with the consequence $t_{0}=-\alpha x$ and

(ii) $x=\beta / \alpha \cdots 1$, with the consequence $t_{0}=-\beta$.

Thus it is

$$
\int_{-1}^{+1} d x \int_{t_{0}}^{+\beta} d t= \begin{cases}\int_{-1}^{+1} d x \int_{-\alpha x}^{+\beta} d t & \text { for } \quad q p \geq 1 \text { or } \alpha \leq \beta \\ \int_{-\beta / \alpha}^{+\beta / \alpha} d x \int_{-\alpha x}^{+\beta} d t+\int_{+\beta / \alpha}^{+1} d x \int_{-\beta}^{+\beta} d t \quad \text { for } \quad q p \leq 1 \text { or } \alpha \geq \beta\end{cases}
$$


With the abbreviations (2.5) and

$$
f(t, x)=\frac{2}{a^{2}-x^{2}} \arctan \frac{t+\alpha x}{\sqrt{\left(1+\alpha^{2}\right)\left(1-x^{2}\right)}},
$$

Eq. (2.6) can be written as (note that $f(-\alpha x, x)=0$ )

$$
\begin{aligned}
& X_{1}=-\int_{0}^{1} d q \int_{1}^{\infty} d p \frac{16 \pi}{\left(p^{2}-q^{2}\right)} \frac{1}{\left(1+q^{2}\right)}\left\{\Theta(q p-1) \int_{-1}^{+1} d x f(\beta, x)\right. \\
& +\Theta(1-q p)\left[\int_{-\beta / \alpha}^{+\beta / \alpha} d x f(\beta, x)+\int_{+\beta / \alpha}^{+1} d x[f(\beta, x)-f(-\beta, x)]\right] .
\end{aligned}
$$

With

$$
\int_{+\beta / \alpha}^{+1} d x(-1) f(-\beta, x)=\int_{+\beta / \alpha}^{+1} d x f(\beta,-x)=\int_{-1}^{-\beta / \alpha} d x f(\beta, x)
$$

the terms for $q p \leq 1$ can be comprised as $\int_{-1}^{+1} d x f(\beta, x)$. Therefore

$$
X_{1}=-\int_{0}^{1} d q \int_{1}^{\infty} d p \int_{-1}^{+1} d x \frac{16 \pi}{p^{2}-q^{2}} \frac{1}{1+q^{2}} f(\beta, x)
$$

The final substitution $p=1 / k$ transforms the region of the last two integrations from the (vertical) stripe $q=0 \cdots 1, p=1 \cdots \infty$ to the more simple unit square $q=0 \cdots 1, k=0 \cdots 1$. With the abbrevations

$$
\alpha=\frac{1-q^{2}}{2 q} \geq 0, \quad \beta=\frac{1-k^{2}}{2 k} \geq 0, \quad a=\frac{1+q^{2} k^{2}}{2 q k} \geq 1
$$

it is:

$$
X_{1}=-\int_{0}^{1} d q \int_{0}^{1} d k \int_{-1}^{+1} d x \frac{16 \pi}{1-q^{2} k^{2}} \frac{1}{1+q^{2}} f(\beta, x) \approx-30.70598 \cdots
$$

The coefficients $\alpha, \beta$, and $a$ of Eq. (2.17) make the integrand of (2.18) functions of $q$ and $k$. Mathematica5.2 17] yields the given figure. It seems to hold

$$
X_{1}=-\pi^{4}\left[\frac{4}{3} \ln 2-5 \frac{\zeta(3)}{\pi^{2}}\right] \approx-30.705985239248893 \cdots
$$

How to derive this analytically? Is this possible with the method of ref. [18]? 


\section{THE INTEGRAL $X_{2}$}

The whole procedure of Sec. II is repeated here step by step. In Eq. (A.11), new variables $\boldsymbol{q}^{\prime}=\left(\boldsymbol{q}_{1}+\boldsymbol{q}_{2}\right) / 2, \boldsymbol{p}^{\prime}=\left(\boldsymbol{q}_{1}-\boldsymbol{q}_{2}\right) / 2$, and $\boldsymbol{s}^{\prime}=\boldsymbol{e}+\left(\boldsymbol{q}_{1}+\boldsymbol{q}_{2}\right) / 2$ lead to

$$
\begin{aligned}
& \boldsymbol{q}_{1}=\boldsymbol{q}^{\prime}+\boldsymbol{p}^{\prime}, \quad\left(\boldsymbol{e}+\boldsymbol{q}_{1}\right)^{2}=\left(\boldsymbol{s}^{\prime}+\boldsymbol{p}^{\prime}\right)^{2}, \quad\left(\boldsymbol{e}+\boldsymbol{q}_{2}\right)^{2}=\left(\boldsymbol{s}^{\prime}-\boldsymbol{p}^{\prime}\right)^{2}, \\
& \boldsymbol{q}_{2}=\boldsymbol{q}^{\prime}-\boldsymbol{p}^{\prime}, \quad\left(\boldsymbol{e}+\boldsymbol{q}_{1}+\boldsymbol{q}_{2}\right)^{2}=\left(\boldsymbol{s}^{\prime}+\boldsymbol{q}^{\prime}\right)^{2}, \quad \boldsymbol{e}^{2}=\left(\boldsymbol{s}^{\prime}-\boldsymbol{q}^{\prime}\right)^{2} .
\end{aligned}
$$

Therefore

$$
\begin{gathered}
X_{2}=\int d^{3} q^{\prime} d^{3} p^{\prime} \frac{2^{3}}{q^{2}-p^{\prime 2}} \frac{1}{\left(\boldsymbol{q}^{\prime}+\boldsymbol{p}^{\prime}\right)^{2}\left(\boldsymbol{q}^{\prime}-\boldsymbol{p}^{\prime}\right)^{2}} \times \\
\times \int \frac{d^{2} s^{\prime}}{\pi} \delta\left(\left(\boldsymbol{s}^{\prime}-\boldsymbol{q}^{\prime}\right)^{2}-1\right) \Theta\left[\left(\boldsymbol{s}^{\prime}+\boldsymbol{q}^{\prime}\right)^{2}-1\right] \Theta\left[1-\left(\boldsymbol{s}^{\prime}+\boldsymbol{p}^{\prime}\right)^{2}\right] \Theta\left[1-\left(\boldsymbol{s}^{\prime}-\boldsymbol{p}^{\prime}\right)^{2}\right] .
\end{gathered}
$$

The next step scales $\boldsymbol{q}^{\prime}, \boldsymbol{p}^{\prime}, \boldsymbol{s}^{\prime}$ with $\lambda=1 / \sqrt{1-s^{\prime 2}}$ according to $\boldsymbol{q}=\lambda \boldsymbol{q}^{\prime}, \boldsymbol{p}=\lambda \boldsymbol{p}^{\prime}, \boldsymbol{s}=\lambda \boldsymbol{s}^{\prime}$ with the consequences

$$
\begin{array}{r}
1-s^{\prime 2}=\frac{1}{1+s^{2}}, \quad d^{2} s^{\prime}=\frac{d^{2} s}{\left(1+s^{2}\right)^{2}}, \\
\delta\left(\left(\boldsymbol{s}^{\prime}-\boldsymbol{q}^{\prime}\right)^{2}-1\right)=\left(1+s^{2}\right) \delta\left(q^{2}-2 \boldsymbol{s} \boldsymbol{q}-1\right), \\
\pm 2 \boldsymbol{s} \boldsymbol{p}+p^{2}<1, \quad 2 \boldsymbol{s} \boldsymbol{q}+q^{2}>1, \quad-2 \boldsymbol{s} \boldsymbol{q}+q^{2}=1,
\end{array}
$$

from which follow $q \geq 1$ and $p \leq 1$ (what makes the energy denominator $q^{2}-p^{2}$ positive) and $\boldsymbol{s} \boldsymbol{q}>0, s \geq \bar{\alpha}$. Thus

$$
\begin{array}{r}
X_{2}=\int_{1}^{\infty} d q \int_{0}^{1} d p \frac{16 \pi}{q^{2}-p^{2}} \int_{-1}^{+1} \frac{d x}{2} \frac{8 q^{2} p^{2}}{\left(p^{2}+q^{2}\right)^{2}-(2 \boldsymbol{q} \boldsymbol{p})^{2}} \times \\
\times \int_{s \geq \bar{\alpha}, \cos \varphi_{q}>0} \frac{d^{2} s}{1+s^{2}} \delta\left(q^{2}-1-2 \boldsymbol{s} \boldsymbol{q}\right) \Theta\left(1-p^{2}-2 \boldsymbol{s} \boldsymbol{p}\right) \Theta\left(1-p^{2}+2 \boldsymbol{s p}\right) .
\end{array}
$$

Here and in the following of Sec. III the abbreviations

$$
\begin{array}{r}
\bar{\alpha}=\frac{q^{2}-1}{2 q} \geq 0, \quad \bar{\beta}=\frac{1-p^{2}}{2 p} \geq 0, \quad \bar{a}=\frac{q^{2}+p^{2}}{2 q p} \geq 1, \\
\frac{t}{s}=\cos \varphi_{p}=\cos \Varangle(\boldsymbol{s}, \boldsymbol{p}), \quad x=\cos \varphi=\cos \Varangle(\boldsymbol{q}, \boldsymbol{p}), \quad y=\cos \varphi_{q}=\cos \Varangle(\boldsymbol{q}, \boldsymbol{s})
\end{array}
$$

are used:

$$
X_{2}=\int_{1}^{\infty} d q \int_{0}^{1} d p \frac{16 \pi}{q^{2}-p^{2}} \int_{-1}^{+1} \frac{d x}{\bar{a}^{2}-x^{2}} \int_{s \geq \bar{\alpha}, \cos \varphi_{q}>0} \frac{s d s d \varphi_{q}}{1+s^{2}} \frac{1}{2 s q} \delta\left(\frac{\bar{\alpha}}{s}-y\right) \Theta(\bar{\beta}-|t|) .
$$


$t$ is introduced to replace $s$ after having done the $\varphi_{q}$ integration. To this purpose the relation between $t$ and $s$ is needed. It follows from $\varphi_{q}+\varphi_{p}=\varphi$ or $\varphi_{p}=\varphi-\varphi_{q}$, therefore $\cos \varphi_{p}=\cos \left(\varphi-\varphi_{q}\right)=\cos \varphi \cos \varphi_{q}+\sin \varphi \sin \varphi_{q}$, what is in terms of $t / s, x$, and $y$ (which equals $+\bar{\alpha} / s$ because of the delta function):

$$
\frac{t}{s}=x \frac{\bar{\alpha}}{s} \pm \sqrt{1-x^{2}} \sqrt{1-\left(\frac{\bar{\alpha}}{s}\right)^{2}} \quad \text { or } \quad(t-\bar{\alpha} x)^{2}=\left(1-x^{2}\right)\left(s^{2}-\bar{\alpha}^{2}\right) .
$$

This has the consequences

$$
\begin{array}{r}
s^{2}-\bar{\alpha}^{2}=\frac{(t-\bar{\alpha} x)^{2}}{1-x^{2}}, \\
\frac{s d s}{1+s^{2}}=\frac{(t-\bar{\alpha} x) d t}{(t-\bar{\alpha} x)^{2}+\left(1+\bar{\alpha}^{2}\right)\left(1-x^{2}\right)} .
\end{array}
$$

With the help of Eq. (3.8) the $\varphi_{q}$ integration yields a function depending on $s$ (respectively on $t$ ). Note that $\cos \varphi_{q}=+\frac{\bar{\alpha}}{s}$ has two solutions $\varphi_{q, 1}$ and $\varphi_{q, 2}$ with $\left|\sin \varphi_{q, 1}\right|=\left|\sin \varphi_{q, 2}\right|=$ $\sqrt{1-\left(\frac{\bar{\alpha}}{s}\right)^{2}}$ :

$$
\begin{aligned}
& \int_{0}^{2 \pi} \frac{d \varphi_{q}}{s}\left[\delta\left(+\sin \varphi_{q, 1} \cdot\left(\varphi_{q}-\varphi_{q, 1}\right)\right)+\delta\left(+\sin \varphi_{q, 2} \cdot\left(\varphi_{q}-\varphi_{q, 2}\right)\right)\right] \\
= & \int_{0}^{2 \pi} \frac{d \varphi_{q}}{s} \frac{\delta\left(\varphi_{q}-\varphi_{q, 1}\right)+\delta\left(\varphi_{q}-\varphi_{q, 2}\right)}{\sqrt{1-\left(\frac{\bar{\alpha}}{s}\right)^{2}}}=\frac{2}{\sqrt{s^{2}-\bar{\alpha}^{2}}}=2 \frac{\sqrt{1-x^{2}}}{|t-\bar{\alpha} x|} .
\end{aligned}
$$

Thus

$$
\begin{aligned}
\int_{s \geq \bar{\alpha}, \cos \varphi_{q}>0} \frac{s d s d \varphi_{q}}{1+s^{2}} \frac{1}{2 s q} \delta\left(\frac{\bar{\alpha}}{s}-y\right) \Theta(\beta-|t|) & =\frac{\sqrt{1-x^{2}}}{q} \int_{t_{0}}^{t_{1}} \frac{\operatorname{sign}(t-\bar{\alpha} x) d t}{(t-\bar{\alpha} x)^{2}+\left(1+\bar{\alpha}^{2}\right)\left(1-x^{2}\right)} \\
& =\left.\frac{2}{1+q^{2}} \arctan \frac{t-\bar{\alpha} x}{\sqrt{\left(1+\bar{\alpha}^{2}\right)\left(1-x^{2}\right)}}\right|_{t_{0}} ^{t_{1}}(3.11)
\end{aligned}
$$

$|t| \leq \bar{\beta}$ contains the upper limit of the $t$-integration as $t \leq \bar{\beta}=: t_{1}$. What concerns its lower limit $t_{0}$, the relation $t \geq-\bar{\beta}$ competes with $t \geq \bar{\alpha} x$, as it follows from Eq. (3.7) for the lower limit $\bar{\alpha}$ of the $s$-integration. This means for the ranges of the $t$ - and $x$-integrations, one has in the (horizontal) stripe $q=1 \cdots \infty, p=0 \cdots 1$ of the $q$-p-plane to distinguish between the two regions, cf. Fig. 4:

(a) region $\mathrm{A}$ with $q p \geq 1$ or $\bar{\alpha} \geq \bar{\beta}$ or $-\bar{\alpha} \leq-\bar{\beta}$, and

(b) region $\mathrm{B}$ with $q p \leq 1$ or $\bar{\alpha} \leq \bar{\beta}$ or $\bar{\alpha} x \leq \bar{\alpha} \leq \bar{\beta}$, hence $t_{0}=\bar{\alpha} x$. 
In the case (a) one has again to distinguish between

(i) $x=-1 \cdots-\bar{\beta} / \bar{\alpha}$, with the consequence $t_{0}=-\bar{\beta}$ and

(ii) $x=-\bar{\beta} / \bar{\alpha} \cdots+\bar{\beta} / \bar{\alpha}$, with the consequence $t_{0}=\bar{\alpha} x$.

Thus it is

$$
\int_{-1}^{+1} d x \int_{t_{0}}^{+\bar{\beta}} d t=\left\{\begin{array}{l}
\int_{-1}^{-\bar{\beta} / \bar{\alpha}} d x \int_{-\bar{\beta}}^{+\bar{\beta}} d t+\int_{-\bar{\beta} / \bar{\alpha}}^{+\bar{\beta} / \bar{\alpha}} d x \int_{\bar{\alpha} x}^{+\bar{\beta}} d t \quad \text { for } \quad q p \geq 1 \text { or } \bar{\alpha} \geq \bar{\beta} \\
\int_{-1}^{+1} d x \int_{\bar{\alpha} x}^{+\bar{\beta}} d t \quad q p \leq 1 \text { or } \bar{\alpha} \leq \bar{\beta}
\end{array}\right.
$$

With the abbreviations (3.5) and with

$$
\bar{f}(t, x)=\frac{2}{\bar{a}^{2}-x^{2}} \arctan \frac{t-\bar{\alpha} x}{\sqrt{\left(1+\bar{\alpha}^{2}\right)\left(1-x^{2}\right)}},
$$

Eq. (3.6) can be written as (note that $\bar{f}(\bar{\alpha} x, x)=0$ )

$$
\begin{gathered}
X_{2}=\int_{1}^{\infty} d q \int_{0}^{1} d p \frac{16 \pi}{q^{2}-p^{2}} \frac{1}{1+q^{2}}\left\{\Theta(1-q p) \int_{-1}^{+1} d x \bar{f}(\bar{\beta}, x)\right. \\
\left.+\Theta(q p-1)\left[\int_{-1}^{-\bar{\beta} / \bar{\alpha}} d x[\bar{f}(\bar{\beta}, x)-\bar{f}(-\bar{\beta}, x)]+\int_{-\bar{\beta} / \bar{\alpha}}^{+\bar{\alpha}} d x \bar{f}(\bar{\beta}, x)\right]\right\} .
\end{gathered}
$$

With the identity

$$
\int_{-1}^{-\bar{\beta} / \bar{\alpha}} d x(-1) \bar{f}(-\bar{\beta}, x)=\int_{-1}^{-\bar{\beta} / \bar{\alpha}} d x \bar{f}(\bar{\beta},-x)=\int_{+\bar{\beta} / \bar{\alpha}}^{+1} d x \bar{f}(\bar{\beta}, x)
$$

the terms for $q p \geq 1$ can be comprised as $\int_{-1}^{+1} d x \bar{f}(\bar{\beta}, x)$. Therefore

$$
X_{2}=\int_{1}^{\infty} d q \int_{0}^{1} d p \int_{-1}^{+1} d x \frac{16 \pi}{q^{2}-p^{2}} \frac{1}{1+q^{2}} \bar{f}(\bar{\beta}, x)
$$

This is similar to Eq. (2.16), but there are also differences. The substitution $q=1 / k$ transforms the region of the last two integrations from the (horizontal) stripe $q=1 \cdots \infty, p=$ $0 \cdots 1$ to the more simple unit square $k=0 \cdots 1, p=0 \cdots 1$. With the abbrevations

$$
\bar{\alpha}=\frac{1-k^{2}}{2 k}, \quad \bar{\beta}=\frac{1-p^{2}}{2 p}, \quad \bar{a}=\frac{1+k^{2} p^{2}}{2 k p}
$$


it is

$$
X_{2}=\int_{0}^{1} d k \int_{0}^{1} d p \int_{-1}^{+1} d x \frac{16 \pi}{1-k^{2} p^{2}} \frac{k^{2}}{1+k^{2}} \bar{f}(\bar{\beta}, x)
$$

Changing finally the notation with $k \rightarrow q$ and $p \rightarrow k$ makes $\bar{\alpha}=\alpha, \bar{\beta}=\beta$, and $\bar{a}=a$, cf. Eq. (2.17). With these identities and with $\bar{f}(t,-x)=f(t, x)$ a further rewriting yields

$$
X_{2}=\int_{0}^{1} d q \int_{0}^{1} d k \int_{-1}^{+1} d x \frac{16 \pi}{1-q^{2} k^{2}} \frac{q^{2}}{1+q^{2}} f(\beta, x) \approx 21.284906 \cdots
$$

This integral differs from Eq. (2.18) 'only' in an additional factor of $-q^{2}$ in the nominator of the integrand. Mathematica5.2 [17] yields the given figure. It seems to hold

$$
X_{2}=\pi^{4}\left[\frac{2}{3} \ln 2-2 \frac{\zeta(3)}{\pi^{2}}\right] \approx 21.284905670516334 \cdots .
$$

How to derive this analytically? Is this possible with the method of ref. [18]?

\section{THE CALCULATION OF $X$}

With Eqs. (2.13), (2.18), (3.18), and with $a, \alpha, \beta$ being defined in Eq. (2.17) the result for $X=X_{1}+X_{2}$ is

$X=-\int_{0}^{1} d q \int_{0}^{1} d k \int_{-1}^{+1} d x \frac{16 \pi}{1-q^{2} k^{2}} \frac{1-q^{2}}{1+q^{2}} \frac{2}{a^{2}-x^{2}} \arctan \frac{\beta+\alpha x}{\sqrt{\left(1+\alpha^{2}\right)\left(1-x^{2}\right)}} \approx-9.42108 \cdots$.

It seems to hold [18, 19]

$$
X=-\pi^{4}\left[\frac{2}{3} \ln 2-3 \frac{\zeta(3)}{\pi^{2}}\right] \approx-9.421079568732553 \cdots .
$$

The final result [20]

$$
\Sigma_{2 \mathrm{x}}=-\frac{\left(\alpha r_{s}\right)^{2}}{4 \pi^{4}} X=e_{2 \mathrm{x}} \quad \text { with } \quad \frac{e_{2 \mathrm{x}}}{\left(\alpha r_{s}\right)^{2}}=\frac{1}{6} \ln 2-\frac{3}{4} \frac{\zeta(3)}{\pi^{2}} \approx 0.0241792
$$

appears in the weak-correlation limit of the Hugenholtz-van Hove (Luttinger-Ward) theorem [14, 15]. Because of $\mu_{2 \mathrm{x}}=e_{2 \mathrm{x}}$ it holds the sum rule $\mu_{2 \mathrm{x}}=\Sigma_{2 \mathrm{x}}$ analogous to $\mu_{\mathrm{x}}=\Sigma_{\mathrm{x}}$. Whether perhaps also the more general expression $\Sigma_{2 \mathrm{x}}(k, \omega)$ can be calculated in a similar way, has to be studied. 


\section{Acknowledgments}

The author thanks M.L. Glasser, U. von Barth, G. Röpke, and R. Zimmermann for valuable hints, U. Saalmann for checking the integrations of Eqs. (2.18) and (3.18) with Mathematica5.2 [17] by independent Fortran calculations, E. Mrosan for a very carefully critical reading the manuscript, F. Tasnadi for his help, and acknowledges P. Fulde for supporting this work.

[1] M.P. Tosi in: N.H. March (Ed.), Electron Correlation in the Solid State, Imperial College Press, 1999, p. 1.

[2] There is a non-vanishing probability of finding also 0 , as well as 2,3 and more electrons in such a Wigner sphere. Such particle number fluctuations in fragments have been studied by P. Ziesche, J. Tao, M. Seidl, and J.P. Perdew, Int. J. Quantum Chem. 77, 819 (2000) with the conclusion 'correlation suppresses fluctuations', cf. also P. Fulde, Electron Correlations in Molecules and Solids, 3rd ed., Springer, Berlin, 1995, p.157.

[3] P. Ziesche, Int J. Quantum Chem. 90, 342 (2002).

[4] P. Ziesche and J. Cioslowski, Physica A 356, 598 (2005). Here it is shown, which peculiarities of $n(k)$ and $S(q)$ caused by RPA leed to $e_{\mathrm{c}} \sim \ln r_{s}$.

[5] R.D. Muiño, I. Nagy, and P.M. Echenique, Phys. Rev. B 72, 075117 (2005).

[6] W. Heisenberg, Z. Naturf. 2a, 185 (1947).

[7] W. Macke, Z. Naturf. 5a, 192 (1950).

[8] M. Gell-Mann and K. Brueckner, Phys. Rev. 106, 364 (1957).

[9] L. Onsager, L. Mittag, and M. J. Stephen, Ann. Physik (Leipzig) 18, 71 (1966). Here the exchange integral for the 3D electron gas has been evaluated analytically. This rather herculean work was generalized and extended to the $d$-dimensional electron gas by M.L. Glasser [10].

[10] M.L. Glasser, J. Comp. Appl. Math. 10, 293 (1984).

[11] M. Vogt, R. Zimmermann, and R. Needs, Phys. Rev. B 69, 045113 (2004).

[12] E. Shirley, Phys. Rev. B 54, 7758 (1996).

[13] C. Fortmann, G. Röpke, and A. Wierling, poster at the workshop "Condensed Matter Theories" (Dresden, 2006); work is in progress. 
[14] N.M. Hugenholtz and L. van Hove, Physica 24, 363 (1958); J.M. Luttinger and J.C. Ward, Phys. Rev. 118, 1417 (1960).

[15] J. Cioslowski and P. Ziesche, submitted; P. Ziesche, submitted.

[16] W.M. Galitskii and A.B. Migdal, Zh. Éksp. Teor. Fiz. 34, 139 (1958) [Sov. Phys. JETP 7, 96 (1958)].

[17] Stephen Wolfram, The Mathematica Book, 5th ed., Wolfram Media/Cambridge University Press, 2005.

[18] M.L. Glasser and G. Lamb, American Mathematical Monthly, to be submitted.

[19] For $X_{1}-X_{2}\left(\right.$ instead of $\left.X_{1}+X_{2}\right)$ it has been shown analytically $X_{1}-X_{2}=-\pi^{4}\left[2 \ln 2-7 \frac{\zeta(3)}{\pi^{2}}\right]$, in agreement with Eqs. (2.19) and (3.19) 18].

[20] This result should be comparable to calculations by E. Shirley [12] if one considers in his Fig. 1 the contribution of the second (exchange) Feynman diagram to the on-shell self-energy $\Sigma(1,1 / 2)$ in the limit $r_{s} \rightarrow 0$, where the effectively screened Coulomb repulsion $W(12)$ is replaced by the bare Coulomb repulsion $\nu(12)$.

\section{APPENDIX A: DERIVATION OF $\Sigma_{2 \mathrm{x}}$}

The one-body Green's function of the non-interacting system (ideal Fermi gas)

$$
G_{0}(k, \omega)=\frac{\Theta(k-1)}{\omega-\frac{1}{2} k^{2}+\mathrm{i} \delta}+\frac{\Theta(1-k)}{\omega-\frac{1}{2} k^{2}-\mathrm{i} \delta}
$$

(with $\Theta(x)=$ Heaviside step function) and $G(k, \omega)$, the one-body Green's function of the fully interacting system, define the self-energy $\Sigma(k, \omega)$ through

$$
G(k, \omega)=G_{0}(k, \omega)+G_{0}(k, \omega) \Sigma(k, \omega) G(k, \omega) .
$$

$\Sigma(k, \omega)$ appears in the Hugenholtz-van Hove theorem (in the Luttinger-Ward form $\mu-\mu_{0}=$ $\Sigma(1, \mu)$ with $\mu=$ chemical potential) [14] and in the Galitskii-Migdal formula [16]

$$
v=\frac{1}{2} \int d(k)^{3} \int \frac{d \omega}{2 \pi \mathrm{i}} \mathrm{e}^{\mathrm{i} \omega \delta} G(k, \omega) \Sigma(k, \omega), \quad \delta_{\rightarrow}^{>} 0 .
$$

$v$ is the potential component of $e$, the total energy per particle. The contour of the $\omega$-integration is to be closed in the upper complex $\omega$-plane. In lowest order it is $\Sigma_{\mathrm{x}}(k)=-\left(1+\frac{1-k^{2}}{2 k} \ln \left|\frac{1+k}{1-k}\right|\right)$. This makes $v_{\mathrm{x}}=-\frac{3}{4} \frac{\alpha r_{s}}{\pi}$, in agreement with $v_{\mathrm{x}}=e_{\mathrm{x}}$, what 
follows from the virial theorem $v=r_{s} \frac{d}{d r_{s}} e$.

From the Feynman diagram for the exchange term of the 2nd-order self-energy it follows

$$
\begin{aligned}
\Sigma_{2 \mathrm{x}}(k, \omega) & =\frac{\left(\alpha r_{s}\right)^{2}}{4 \pi^{4}} \int \frac{d^{3} q_{1} d^{3} q_{2}}{q_{1}^{2} q_{2}^{2}} \int \frac{d \eta_{1} d \eta_{2}}{(2 \pi \mathrm{i})^{2}} \times \\
& \times G_{0}\left(\left|\boldsymbol{k}+\boldsymbol{q}_{2}\right|, \omega+\eta_{2}\right) G_{0}\left(\left|\boldsymbol{k}+\boldsymbol{q}_{1}+\boldsymbol{q}_{2}\right|, \omega+\eta_{1}+\eta_{2}\right) G_{0}\left(\left|\boldsymbol{k}+\boldsymbol{q}_{1}\right|, \omega+\eta_{1}\right)
\end{aligned}
$$

Use of (A.1) yields

$$
\begin{aligned}
\Sigma_{2 \mathrm{x}}(k, \omega)=-\frac{\left(\alpha r_{s}\right)^{2}}{4 \pi^{4}} \int \frac{d^{3} q_{1} d^{3} q_{2}}{q_{1}^{2} q_{2}^{2}} & {\left[\frac{\Theta\left(\left|\boldsymbol{k}+\boldsymbol{q}_{1}+\boldsymbol{q}_{2}\right|-1\right) \Theta\left(1-\left|\boldsymbol{k}+\boldsymbol{q}_{1}\right|\right) \Theta\left(1-\left|\boldsymbol{k}+\boldsymbol{q}_{2}\right|\right)}{\omega-\frac{1}{2} k^{2}+\boldsymbol{q}_{1} \cdot \boldsymbol{q}_{2}-\mathrm{i} \delta}\right.} \\
+ & \left.\frac{\Theta\left(1-\left|\boldsymbol{k}+\boldsymbol{q}_{1}+\boldsymbol{q}_{2}\right|\right) \Theta\left(\left|\boldsymbol{k}+\boldsymbol{q}_{1}\right|-1\right) \Theta\left(\left|\boldsymbol{k}+\boldsymbol{q}_{2}\right|-1\right)}{\omega-\frac{1}{2} k^{2}+\boldsymbol{q}_{1} \cdot \boldsymbol{q}_{2}+\mathrm{i} \delta}\right] .
\end{aligned}
$$

One may check this expression by using it in the Galitskii-Migdal formula (A.3). Its lhs is known from the virial theorem as $v_{2 \mathrm{x}}=2 e_{2 \mathrm{x}}$ with $e_{2 \mathrm{x}}=$ energy in second order of exchange, calculated by Onsager et al. 9]. Its rhs gives with Eqs. (A.1) and (A.5)

$$
\begin{aligned}
\mathrm{rhs}=- & \frac{3\left(\alpha r_{s}\right)^{2}}{(2 \pi)^{5}}\left[\int \frac{d^{3} k d^{3} q_{1} d^{3} q_{2}}{q_{1}^{2} q_{2}^{2}} \frac{\Theta(1-k) \Theta\left(1-\left|\boldsymbol{k}+\boldsymbol{q}_{1}+\boldsymbol{q}_{2}\right|\right) \Theta\left(\left|\boldsymbol{k}+\boldsymbol{q}_{1}\right|-1\right) \Theta\left(\left|\boldsymbol{k}+\boldsymbol{q}_{2}\right|-1\right)}{\boldsymbol{q}_{1} \cdot \boldsymbol{q}_{2}+\mathrm{i} \delta}\right. \\
& \left.+\int \frac{d^{3} k d^{3} q_{1} d^{3} q_{2}}{q_{1}^{2} q_{2}^{2}} \frac{\Theta(k-1) \Theta\left(\left|\boldsymbol{k}+\boldsymbol{q}_{1}+\boldsymbol{q}_{2}\right|-1\right) \Theta\left(1-\left|\boldsymbol{k}+\boldsymbol{q}_{1}\right|-1\right) \Theta\left(1-\left|\boldsymbol{k}+\boldsymbol{q}_{2}\right|\right)}{\boldsymbol{q}_{1} \cdot\left(-\boldsymbol{q}_{2}\right)+\mathrm{i} \delta}\right] .
\end{aligned}
$$

It is easy to show with the help of the substitutions $\boldsymbol{q}_{1} \rightarrow \boldsymbol{q}_{1}^{\prime}, \boldsymbol{q}_{2} \rightarrow-\boldsymbol{q}_{2}^{\prime}, \boldsymbol{k} \rightarrow-\left(\boldsymbol{k}^{\prime}+\boldsymbol{q}_{1}^{\prime}\right)$ that the second term equals the first one. Thus

$$
\begin{aligned}
\text { Re rhs } & =-2 \frac{3\left(\alpha r_{s}\right)^{2}}{(2 \pi)^{5}} \int \frac{d^{3} k d^{3} q_{1} d^{3} q_{2}}{q_{1}^{2} q_{2}^{2}} \frac{P}{\boldsymbol{q}_{1} \cdot \boldsymbol{q}_{2}} \times \\
& \times \Theta(1-k) \Theta\left(1-\left|\boldsymbol{k}+\boldsymbol{q}_{1}+\boldsymbol{q}_{2}\right|\right) \Theta\left(\left|\boldsymbol{k}+\boldsymbol{q}_{1}\right|-1\right) \Theta\left(\left|\boldsymbol{k}+\boldsymbol{q}_{2}\right|-1\right)=2 e_{2 \mathrm{x}} .
\end{aligned}
$$

$P$ means the Cauchy principle value. This is in agreement with the above mentioned relation. That $e_{2 x}$ of Eq. (A.7) really agrees with the integral calculated by Onsager et al. 9] follows from the substitutions $\boldsymbol{k} \rightarrow \boldsymbol{k}_{1}, \boldsymbol{q}_{1} \rightarrow \boldsymbol{q}, \boldsymbol{q}_{2} \rightarrow-\left(\boldsymbol{k}_{1}+\boldsymbol{k}_{2}+\boldsymbol{q}\right)$. From Eq. (A.5) also follows $n_{2 \mathrm{x}}(k)$, the second-order-in-exchange contribution to the momentum distribution. It is again easy to derive the well-known asymptotics $n_{2 \mathrm{x}}(k \rightarrow \infty)=-\frac{4}{9 \pi^{2}} \frac{\left(\alpha r_{s}\right)^{2}}{k^{8}}$. 
After this control of $\Sigma_{2 \mathrm{x}}(k, \omega)$, the formula for $\Sigma_{2 \mathrm{x}}=\operatorname{Re} \Sigma_{2 \mathrm{x}}(1,1 / 2)$ follows from Eq. (A.5) as $\Sigma_{2 \mathrm{x}}=-\frac{\left(\alpha r_{s}\right)^{2}}{4 \pi^{4}}\left(X_{1}+X_{2}\right)$, where $X_{1,2}$ mean the integrals

$$
\begin{aligned}
& X_{1}=\int d^{3} q_{1} d^{3} q_{2} \frac{P}{\boldsymbol{q}_{1} \cdot \boldsymbol{q}_{2}} \frac{1}{q_{1}^{2} q_{2}^{2}} \Theta\left[1-\left(\boldsymbol{e}+\boldsymbol{q}_{1}+\boldsymbol{q}_{2}\right)^{2}\right] \Theta\left[\left(\boldsymbol{e}+\boldsymbol{q}_{1}\right)^{2}-1\right] \Theta\left[\left(\boldsymbol{e}+\boldsymbol{q}_{2}\right)^{2}-1\right] \\
& X_{2}=\int d^{3} q_{1} d^{3} q_{2} \frac{P}{\boldsymbol{q}_{1} \cdot \boldsymbol{q}_{2}} \frac{1}{q_{1}^{2} q_{2}^{2}} \Theta\left[\left(\boldsymbol{e}+\boldsymbol{q}_{1}+\boldsymbol{q}_{2}\right)^{2}-1\right] \Theta\left[1-\left(\boldsymbol{e}+\boldsymbol{q}_{1}\right)^{2}\right] \Theta\left[1-\left(\boldsymbol{e}+\boldsymbol{q}_{2}\right)^{2}\right] .
\end{aligned}
$$

They contain $\boldsymbol{q}_{1} \cdot \boldsymbol{q}_{2}$ as the energy denominator. $1 / q_{1,2}^{2}$ arises from the Coulomb repulsion and the remainder is due to the Pauli principle. $\boldsymbol{e}$ is a unit vector. Note that $\boldsymbol{q}_{1} \cdot \boldsymbol{q}_{2}<0$ for $X_{1}$, because of $\left(2 \boldsymbol{e}+\boldsymbol{q}_{1}\right) \cdot \boldsymbol{q}_{1}>0$ and $\left(2 \boldsymbol{e}+\boldsymbol{q}_{2}\right) \cdot \boldsymbol{q}_{2}>0$ in combination with $\left(2 \boldsymbol{e}+\boldsymbol{q}_{1}\right) \cdot \boldsymbol{q}_{1}+$ $\left(2 \boldsymbol{e}+\boldsymbol{q}_{2}\right) \cdot \boldsymbol{q}_{2}+2 \boldsymbol{q}_{1} \cdot \boldsymbol{q}_{2}<0$. This latter inequality enforces $\boldsymbol{q}_{1} \cdot \boldsymbol{q}_{2}<0$. Thus $X_{1}<0$. It follows similarly $X_{2}>0$. The integrals $X_{1,2}$ do not depend on $\boldsymbol{e}$. Therefore application of $\hat{O}=\int d^{3} e /(4 \pi) 2 \delta\left(\boldsymbol{e}^{2}-1\right)$ does not change them (notice $2 \delta\left(\boldsymbol{e}^{2}-1\right)=\delta(e-1)$ ). Following Onsager et al. 9], $\boldsymbol{e}$ is resolved into its components perpendicular to the $\boldsymbol{q}_{1}-\boldsymbol{q}_{2}$-plane $\boldsymbol{e}_{\perp}$, and in the plane $\boldsymbol{e}_{\|}: \hat{O}=\int d e_{\perp} / 2 \int d^{2} e_{\|} /(2 \pi) 2 \delta\left(e_{\|}^{2}+e_{\perp}^{2}-1\right)$. The integration over $\boldsymbol{e}_{\perp}$ may be done immediately by means of a change in scale: $\boldsymbol{q}_{1}=\tilde{\boldsymbol{q}}_{1} \sqrt{1-e_{\perp}^{2}}, \boldsymbol{q}_{2}=\tilde{\boldsymbol{q}}_{2} \sqrt{1-e_{\perp}^{2}}$, $\boldsymbol{e}_{\|}=\tilde{\boldsymbol{e}}_{\|} \sqrt{1-e_{\perp}^{2}}$. The results are (denoting $\tilde{\boldsymbol{e}}_{\|}$as $\boldsymbol{e}$ and deleting also all the other tildes for simplicity)

$$
\begin{aligned}
& X_{1}= \int d^{3} q_{1} d^{3} q_{2} \frac{P}{\boldsymbol{q}_{1} \cdot \boldsymbol{q}_{2}} \frac{1}{q_{1}^{2} q_{2}^{2}} \int \frac{d^{2} e}{2 \pi} 2 \delta\left(\boldsymbol{e}^{2}-1\right) \times \\
& \times \Theta\left[1-\left(\boldsymbol{e}+\boldsymbol{q}_{1}+\boldsymbol{q}_{2}\right)^{2}\right] \Theta\left[\left(\boldsymbol{e}+\boldsymbol{q}_{1}\right)^{2}-1\right] \Theta\left[\left(\boldsymbol{e}+\boldsymbol{q}_{2}\right)^{2}-1\right], \\
& X_{2}=\int d^{3} q_{1} d^{3} q_{2} \frac{P}{\boldsymbol{q}_{1} \cdot \boldsymbol{q}_{2}} \frac{1}{q_{1}^{2} q_{2}^{2}} \int \frac{d^{2} e}{2 \pi} 2 \delta\left(\boldsymbol{e}^{2}-1\right) \times \\
& \times \Theta\left[\left(\boldsymbol{e}+\boldsymbol{q}_{1}+\boldsymbol{q}_{2}\right)^{2}-1\right] \Theta\left[1-\left(\boldsymbol{e}+\boldsymbol{q}_{1}\right)^{2}\right] \Theta\left[1-\left(\boldsymbol{e}+\boldsymbol{q}_{2}\right)^{2}\right] .
\end{aligned}
$$

Whereas the two terms of $\Sigma_{2 \mathrm{x}}(k, \omega)$, cf. (A.6) each contributes $e_{2 \mathrm{x}}$ to $v_{2 \mathrm{x}}$ (thus $v_{2 \mathrm{x}}=2 e_{2 \mathrm{x}}$ ) as shown above, cf. (A.7), $X_{1}$ and $X_{2}$ contribute different values to $\Sigma_{2 \mathrm{x}}(1,1 / 2)$ as shown in Secs. II and III. 


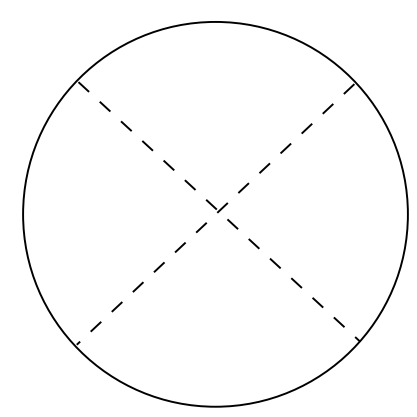

FIG. 1: The Feynman diagram of $e_{2 \mathrm{x}}$, analytically calculated by Onsager et al. 9 ].

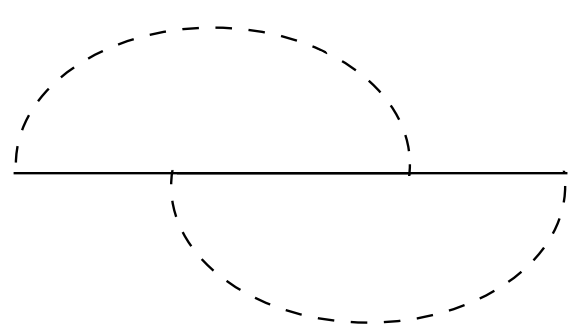

FIG. 2: The Feynman diagram of $\Sigma_{2 \mathrm{x}}(k, \omega)$, (semi)analytically calculated in this paper. 

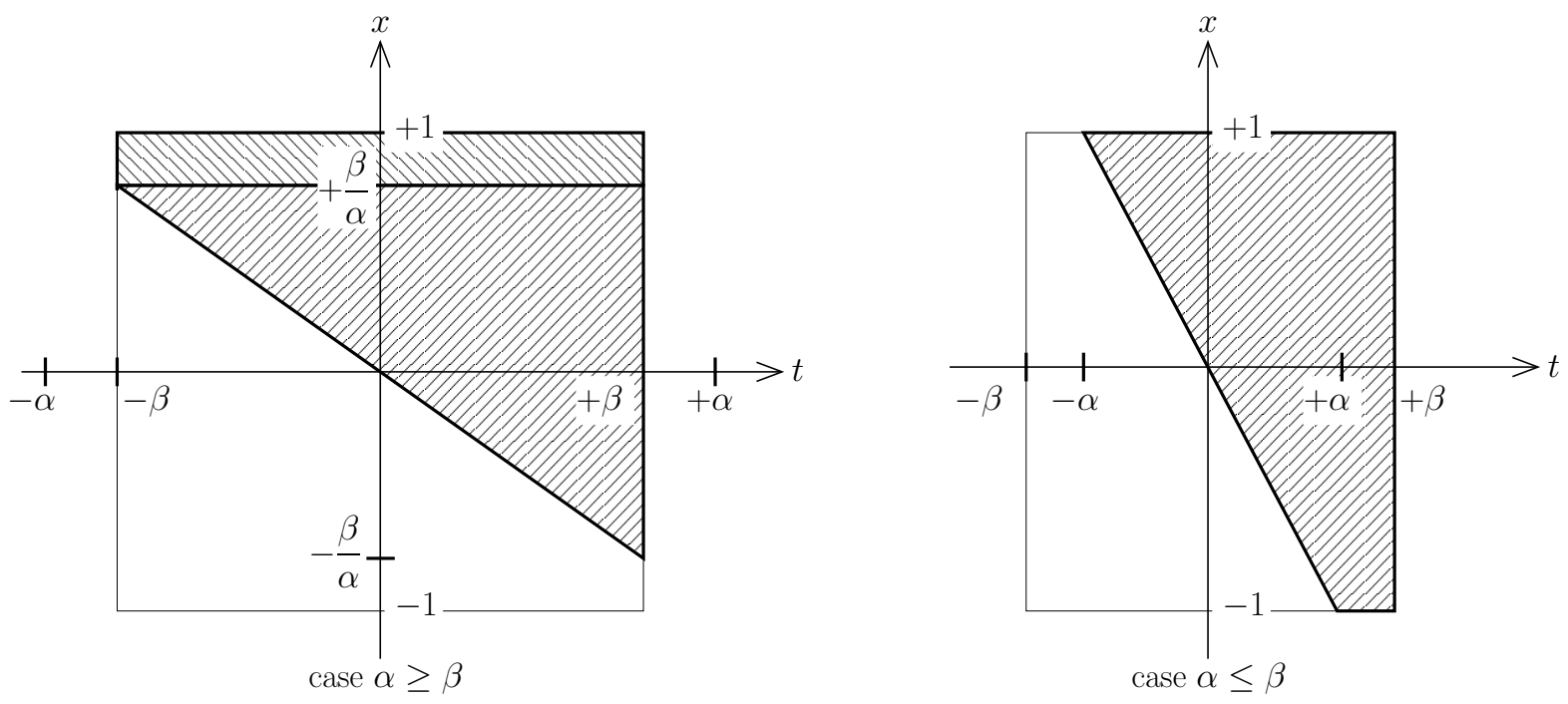

FIG. 3: The dashed area is the region of integration described by Eq. (2.12).
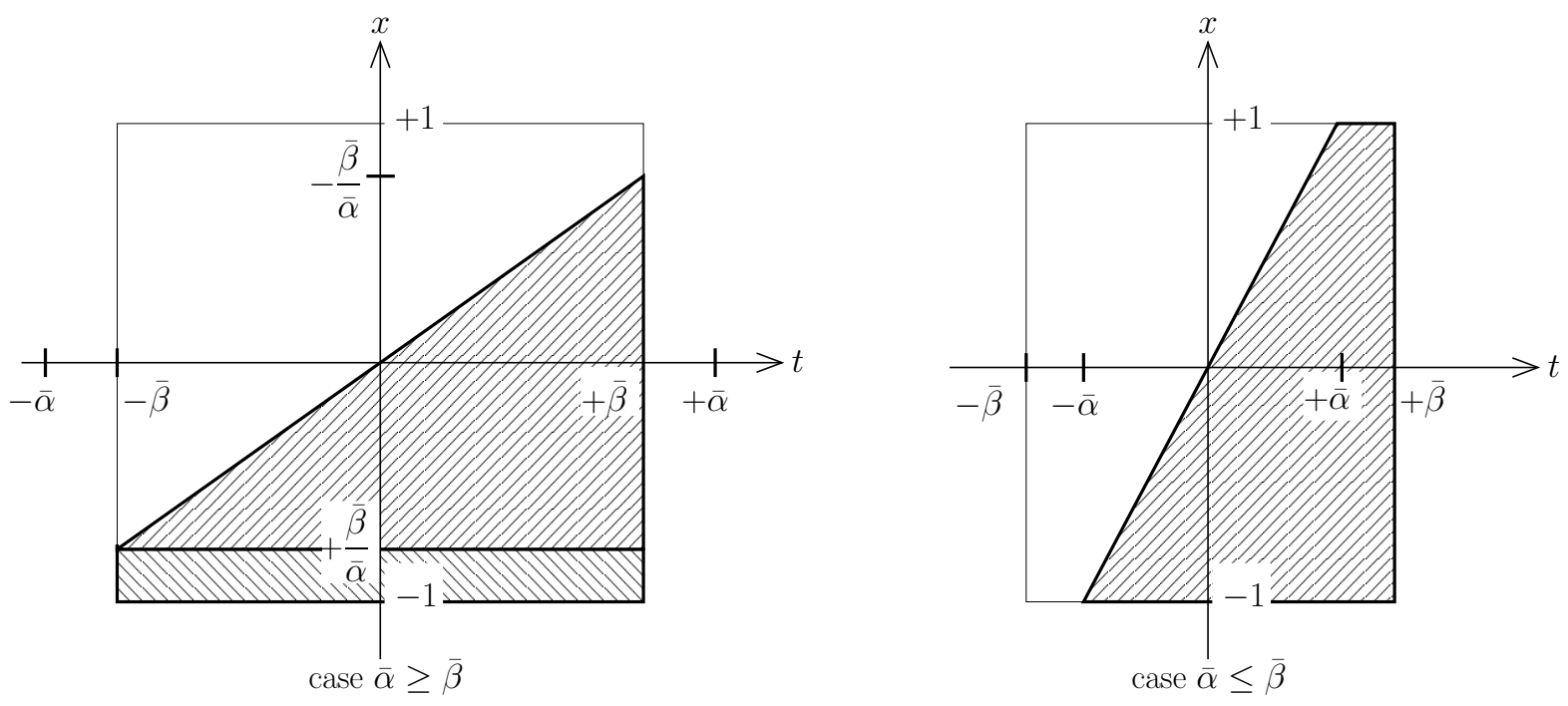

FIG. 4: The dashed area is the region of integration described by Eq. (3.12). 